\title{
Hypertrophic pachymeningitis secondary to Erdheim-Chester disease
}

Keywords: erdheim chesterdisease, hypertrophic pachymeningitis

\section{Introduction}

Erdheim-Chester disease (ECD) is a rare, systemic, nonLangerhans form of histiocytosis characterized by foamy macrophage infiltration. The clinical manifestations can be multi-organ. The cortical involvement of the long bones is characteristic, presenting symmetric metadiaphyseal osteoesclerosis. Infiltration of the central nervous system (CNS) and cardiovascular system constitute an independent factor of poor prognosis. ${ }^{1} \mathrm{CNS}$ involvement is observed in approximately $40 \%$ of patients, with $40 \%$ to $50 \%$ of them suffering from peripheral neuropathy, cognitive impairment, and ataxia. Hypertrophic pachymeningitis is a rare complication of ECD. ${ }^{2}$ In this work, we examine the case of a female patient with ECD presenting with progressive neurological deterioration and hypertrophic pachymeningitis.

\section{Clinical case}

Our patient is a 56-year-old woman with no relevant medical history who consulted for a 6-year history that started with facial dyskinesia and depressive syndrome. She was treated with escitalopram and olanzapine, obtaining a moderate response to treatment for 3 years. Polydipsia, polyuria, apathy, abulia, gait instability, dizziness, visual and auditory hallucinations, swallowing disorder and delirium were added. She evolved with deterioration of the general state, prostration, loss of sphincter control, apathy, prosopagnosia and prolonged fever syndrome. On physical examination, the patient had a posture in flexion, was alert, had bradypsychia and dysprosexia, and was partially oriented in person and disoriented in time and space. She also had muscular atrophy and pyramidal syndrome in 4 limbs. Brain magnetic resonance imaging (MRI) showed marked dural reinforcement at the tentorial level with a diagnosis of hypertrophic pachymeningitis. PET-CT showed an increase in uptake in the tentorium, the abdominal aorta and the left upper pulmonary lobe. Increased bilateral uptake was also observed distally in both femurs and proximal in both tibiae. In the skull in the right frontal bone, the left jugular foramen and in the cervical spine at the $\mathrm{C} 4$ level (Figure 1).

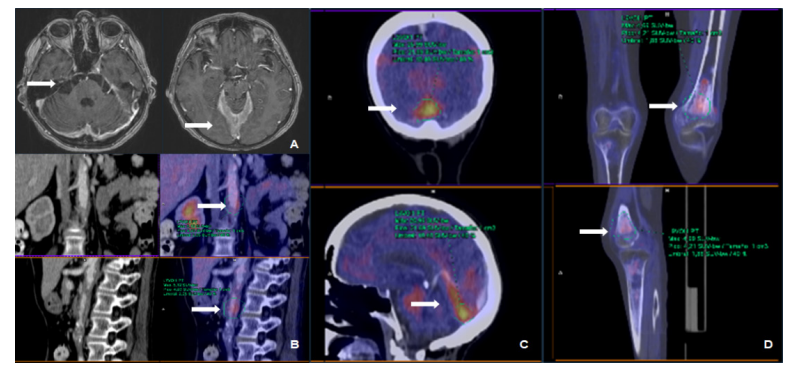

Figure I A) Brain MRI with dural fibroinflammatory involvement, findings consistent with hypertrophic pachymeningitis. B) PET-CT with a thickened and irregular parietal appearance of the abdominal aorta, with a slight diffuse increase in FDG fixation. C) PET-CT with increased metabolism in the tentorium in supravermian topography attributable to meningeal involvement. D) PET-CT with alteration of the usual bone trabeculate of the distal femoral diaphysis and proximal tibiae with a slight increase in the concentration of the FDG, observing irregular radiolucent areas and others with a sclerous appearance.
Volume 10 Issue 6 - 2020

\author{
Marianela López Armaretti, 'Varela Lucia,' \\ Mariano Murillo, ${ }^{2}$ Miguel Sassano, ${ }^{2}$ Cristina \\ Zurrú \\ 'Hospital Italiano de Buenos Aires, Neurology Service, Ciudad \\ Autónoma de Buenos Aires, Argentina \\ ${ }^{2}$ Hospital Italiano de Buenos Aires, Internal Medicine Service, \\ Ciudad Autónoma de Buenos Aires, Argentina
}

\begin{abstract}
Correspondence: Marianela Lopez Armaretti, Peron 4190 Hospital Italiano de Buenos Aires, Servicio de Neurología. Primer Piso, Código Postal, Ciudad Autónoma de Buenos Aires, Argentina, Email marianela.lopez@hospitalitaliano.org.ar
\end{abstract}

Received: September 0I, 2020 | Published: November 24, 2020

The laboratory showed anemia and a slight increase in the of globular sedimentation rate. Infection with HIV, VDRL, hepatitis $\mathrm{B}$ and $\mathrm{C}$, Chagas and blood cultures were negative. Determination of anti-neuronal antibodies (AMPHYPHISINE, CV2 / CRMP5, PNMA2 (Ma2 / TA), YO, HU, Recoverina, SOX 1, TITINA, ZIC4, GAD65, TR DNER, NMDA, AMPA1 and 2, CASPR2, LGI1, GABA B1 / 2, SI) and the onconeuronal antibodies were negative. The following inflammatory diseases were ruled out: IgG4 deposition, celiac disease and connective tissue diseases. Cerebrospinal fluid (CSF) analysis showed few lymphocytes and macrophages. Flow cytometry without atypical cells. VDRL, Ziehl Neelsen staining, PCR for Mycobacterium tuberculosis, PCR for herpes virus, and cultures for typical and atypical bacteria were all negative. CD68 was positive and cytokeratin AE1-AE3 was negative.

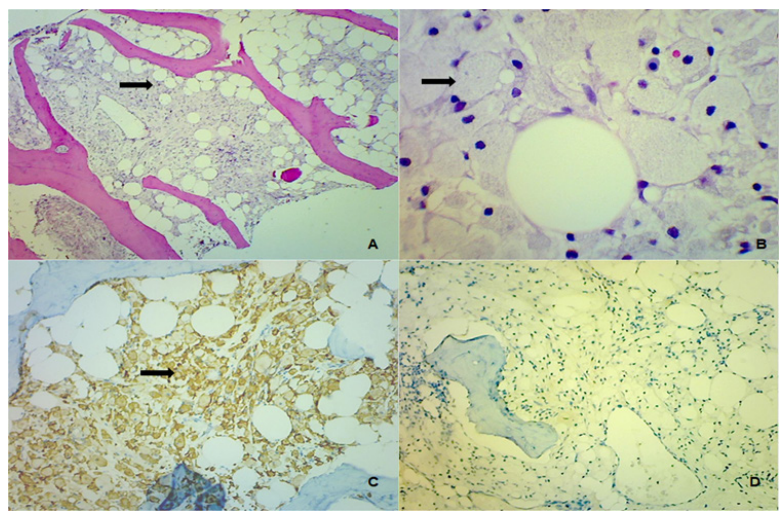

Figure 2 Bone biopsy. A and B) Hematoxylin-eosin stain: Medullary spaces infiltrated by histiocytes (arrow) with a wide, clear and foamy cytoplasm, surrounded by fat cells characteristic of bone tissue. C) CDI63 positive. D) CDIa negative. 
Upon suspicion of ECD, a bone biopsy was performed, finding a foamy macrophage infiltrate and medullary fibrosis with CD68 and CD163 positive, S100 and CD1a were negative by immunohistochemistry. The BRAFV600E mutation was negative (Figure 2). The neurological compromise was progressive. The MRI showed an extension of the pachymeningitis. The presence of CD68 positive macrophages in CSF, foamy macrophages in bone biopsy with CD68 and CD163 positive and S100 negative and hypercaptive foci in long bones on PET-CT, confirm the diagnosis of ECD. She received treatment with interferon alfa $2 \mathrm{~b} 3,000,000$ IU subcutaneously on a three time a week schedule. After 3 months of follow-up, there were no clinical changes.

\section{Discussion}

ECD is a rare form of non-Langerhans cell histiocytosis which generally occurs between the ages of 40 and 70 with some predilection for men. ${ }^{2.3}$ Neuro-ECD is often confused with other diseases including progressive multiple sclerosis, IgG4 disease, CNS neurosurgery, CNS vasculitis, or adrenoleukodystrophy. Glucocorticoids can sometimes provide some temporary improvement. In patients with a poor response to lymphocyte-targeted immunotherapy, ECD should be considered. In X-rays, ECD lesions may misleadingly look like sarcoid, lymphoma, atypical multiple sclerosis, astrocytoma, and leukoencephalopathy. ECD lesions are typically multifocal, variable in size and with well-defined borders; they seldom improve and rarely result in a significant mass effect or edema. ${ }^{4}$ Hypertrophic pachymeningitis is a rare manifestation. The images in long bones and various organs in the PET-CT are useful to suspect the diagnosis and choose the site to biopsy. ${ }^{5}$

In the prospective study that included the most patients (multicenter observation cohort of 53 patients), it was found that the diagnostic delay was an average of 89 months, while our patient was diagnosed with 75 months of evolution. The comparison of the survival curves of this study revealed significantly better survival in the patients who received PEGylated interferon $\alpha$ and / or $\alpha$, so it was decided to start this treatment in our patient. ${ }^{6}$

In conclusion, neurologists play a critical role in identifying and monitoring ECD and other histiocytic disorders, because patients may present with neurologic symptoms, isolated neurologic disease occurs, and neurologic involvement portends a poorer prognosis.
Furthermore, identification of ECD can spare patients morbidity associated with immunotherapies directed toward other diseases and ineffective therapies. It is therefore concluded that the role played by neurologists in the identification and monitoring of ECD as well as other histiocytic disorders is paramount since patients may exhibit neurological symptoms, an isolated neurological disease may occur, and a poorer prognosis is expected when neurological involvement is observed. In addition, adequate ECD detection may spare patients from morbidities associated with both immunotherapies aimed at fighting other diseases and ineffective therapies.

\section{Acknowledgments}

None.

\section{Conflicts of interest}

The authors declare no conflicts of interest.

\section{References}

1. Cives M, Simone V, Rizzo F, et al. Erdheim - Chester disease: A systematic review. Crit Rev Oncol Hematol. 2015;95(1):1-11.

2. Diamond EL, Dagna L, Hyman DM, et al. Consensus guidelines for the diagnosis and clinical management of Erdheim-Chester disease. Blood. 2014;124(4):483-92.

3. Cavalli G, Guglielmi B, Berti A, et al. The multifaceted clinical presentations and manifestations of Erdheim-Chester disease: comprehensive review of the literature and of 10 new cases. Ann Rheum Dis. 2013;72(10):1691-1695.

4. Sedrak P, Ketonen L,Hou P, et al. Erdheim-Chester disease of the central nervous system: new manifestations of a rare disease. AJNR Am J Neuroradiol. 2011;32(11):2126-2131.

5. Goyal G, Young JR, Koster MJ, et al. Mayo Clinic Histiocytosis Working Group. The Mayo Clinic Histiocytosis Working Group Consensus Statement for the Diagnosis and Evaluation of AdultPatients With Histiocytic Neoplasms: Erdheim-Chester Disease, Langerhans Cell Histiocytosis, and Rosai-Dorfman Disease. Mayo Clin Proc. 2019;94(10):2054-2071.

6. Arnaud L, Hervier B, Néel A, et al. CNS involvement and treatment with interferon- $\alpha$ are independent prognostic factors in ErdheimChester disease: a multicenter survival analysis of 53 patients. Blood. 2011;117(10):2778-2782. 